\title{
A COMBINATORIAL THEOREM WITH AN APPLICATION TO LATIN RECTANGLES
}

\author{
H. J. RYSER
}

1. Introduction. In the present paper a study is made of matrices of $r$ rows and $n$ columns, composed entirely of zeros and ones, with exactly $k$ ones in each row. The problem considered is that of adjoining $n-r$ rows of zeros and ones to obtain a square matrix with exactly $k$ ones in each row and in each column. In $\$ 2$ it is shown that the obvious necessary conditions for the adjunction of $n-r$ rows are also sufficient. The theorem of $\$ 2$ has an immediate application to the study of latin squares, and yields in $\$ 3$ a generalization of the basic existence theorem of Marshall Hall [2]. ${ }^{1}$

\section{A combinatorial theorem.}

Theorem 1. Let $A$ be a matrix of $r$ rows and $n$ columns, composed entirely of zeros and ones, where $1 \leqq r<n$. Let there be exactly $k$ ones in each row, and let $N(i)$ denote the number of ones in the ith column of $A$. If, for each $i=1,2, \cdots, n$,

$$
k-(n-r) \leqq N(i) \leqq k,
$$

then $n-r$ rows of zeros and ones may be adjoined to $A$ to obtain a square matrix with exactly $k$ ones in each row and in each column.

The proof is by mathematical induction. Let $t$ denote the number of columns of $A$ with $N(i)<k$. Then $n-t$ denotes the number of columns of $A$ with $N(i)=k$, and consequently $k r=N(1)+\cdots$ $+N(n) \geqq(n-t) k+(k-(n-r)) t$. Thus $k(r-n) \geqq t(r-n)$, whence $t \geqq k$.

Next let $p$ denote the number of columns of $A$ with $N(i)=k$ $-(n-r)$. Then $n-p$ denotes the number of columns with $N(i)$ $>k-(n-r)$. Consequently $k r=N(1)+\cdots+N(n) \leqq p(k-(n-r))$ $+(n-p) k$, whence $k(r-n) \leqq p(r-n)$ and $p \leqq k$.

We now adjoin to $A$ a row consisting of $k$ ones and $n-k$ zeros. Since $t \geqq k$, there are at least $k$ positions where ones may be inserted so that the resulting $(r+1)$-rowed matrix will have at most $k$ ones in each column. Moreover, since $p \leqq k$, the ones may be inserted in all of those columns with $N(i)=k-(n-r)$. In the resulting $(r+1)$ rowed matrix, let $M(i)$ denote the number of ones in the $i$ th column.

Presented to the Society, November 25, 1950; received by the editors September 16, 1950.

1 Numbers in brackets refer to the references at the end of the paper 
Because of the structure of the adjoined row, it is clear that

$$
k-(n-(r+1)) \leqq M(i) \leqq k .
$$

The process may be continued inductively, and the resulting square matrix possesses $k$ ones in each row and column.

A rectangular matrix $L$ composed of zeros and ones is called a permutation matrix provided that it satisfies the equation $L L^{T}=I$, where $L^{T}$ is the transpose of $L$ and $I$ is the identity matrix. Let $A$ be a square matrix of zeros and ones, with exactly $k$ ones in each row and in each column. A classical theorem of König asserts that

$$
A=L_{1}+L_{2}+\cdots+L_{k}
$$

where the $L_{i}$ are permutation matrices [5]. Actually König's theorem is a special case of P. Hall's theorem on the distinct representatives of subsets [4]. The latter theorem has been the subject of the recent investigations of Everett and Whaples [1], and Marshall Hall [3].

Corollary. For the matrix $A$ of Theorem 1, $A=L_{1}+L_{2}+\cdots+L_{k}$, where the $L_{i}$ are permutation matrices.

The corollary follows immediately upon and application of Theorem 1 and König's theorem.

3. The application to latin rectangles. A latin rectangle of order $r$ by $s$ based upon the integers $1,2, \cdots, n$ is defined as an array of $r$ rows and $s$ columns formed from the integers $1,2, \cdots, n$ in such a way that the integers in each row and in each column are distinct. The latin rectangle is said to be extendible to an $n$ by $n$ latin square provided that it is possible to adjoin $n-s$ columns and $n-r$ rows in such a way that the resulting array is an $n$ by $n$ latin square. By utilizing the theory of distinct representatives of subsets, Marshall Hall has shown that every $r$ by $n$ latin rectangle may be extended to an $n$ by $n$ latin square [2].

THEOREM 2. Let $T$ be an $r$ by s latin rectangle based upon the integers $1,2, \cdots, n$. Let $N(i)$ denote the number of times that the integer $i$ occurs in $T$. A necessary and sufficient condition in order that $T$ may be extended to an $n$ by $n$ latin square is that for each $i=1,2, \cdots, n$,

$$
N(i) \geqq r+s-n \text {. }
$$

Let $T_{i}$ denote the set of $s$ integers formed from the $i$ th row of $T$. Let $S_{i}$ denote the set of the $k=n-s$ integers among $1,2, \cdots, n$ which are not in $T_{i}$, and let $M(i)$ denote the number of times that the integer $i$ occurs among the sets $S_{1}, S_{2}, \cdots, S_{r}$. 
Now if $T$ is extendible to a latin square, then the integer $i$ cannot occur among the sets $S_{1}, S_{2}, \cdots, S_{r}$ more than $k=n-s$ times. Hence $M(i) \leqq n-s$. But $N(i)+M(i)=r$, whence $N(i) \geqq r+s-n$. Thus the condition of the theorem is a necessary one.

To prove the sufficiency we form from the sets $S_{i}$ a matrix $A$ of order $r$ by $n$, composed of zeros and ones. Let $S_{i}$ be composed of the integers $i_{1}, i_{2}, \cdots, i_{k}$. In the $i$ th row of $A$ insert ones in columns $i_{1}, i_{2}, \cdots, i_{k}$, and zeros elsewhere in this row. The matrix $A$ has then exactly $k$ ones in each row, and $M(i)$ is now the sum of the $i$ th column of $A$. By hypothesis $N(i)=r-M(i) \geqq r+s-n$, so that for $i$ $=1,2, \cdots, n, M(i) \leqq k$. Since $T$ is an $r$ by $s$ latin rectangle, $N(i) \leqq s$, whence $k-(n-r) \leqq M(i)$. By the corollary of Theorem 1, it now follows that $A=L_{1}+L_{2}+\cdots+L_{k}$, where the $L_{t}$ are rectangular permutation matrices. Let the one in row $j$ of $L_{t}$ occur in column $t_{j}$. From the integers $t_{j}$ form the $k$ sets $\left(t_{1}, t_{2}, \cdots, t_{r}\right)$, each containing $r$ distinct integers. These sets may now be adjoined to $T$ to obtain a latin rectangle of order $r$ by $n$. The latter may then be extended to an $n$ by $n$ latin square as in [2]. This does not differ essentially from completing the transposed $n$ by $r$ latin rectangle to an $n$ by $n$ latin square by the method already indicated, the condition on $N(i)$ being then trivially satisfied.

\section{REFERENCES}

1. C. J. Everett and G. Whaples, Representations of sequences of sets, Amer. J. Math. vol. 71 (1949) pp. 287-293.

2. Marshall Hall, An existence theorem for latin squares, Bull. Amer. Math. Soc. vol. 51 (1945) pp. 387-388.

3. - Distinct representatives of subsets, Bull. Amer. Math. Soc. vol. 54 (1948) pp. 922-926. 26-30.

4. P. Hall, On representatives of subsets, J. London Math. Soc. vol. 10 (1935) pp.

5. Dénes König, Über Graphen und ihre Anwendung auf Determinantentheorie und Mengenlehre, Math. Ann. vol. 77 (1916) pp. 453-465.

Ohio State University 